Cahiers $d u$ MONDE RUSSE

\section{Cahiers du monde russe}

Russie - Empire russe - Union soviétique et États indépendants

$56 / 4 \mid 2015$

Médiateurs d'empire en Asie centrale (1820-1928)

\title{
Maxim D. Shrayer, I Saw It, Ilya Selvinsky and the Legacy of Bearing Witness to the Shoah
}

\section{Catherine Depretto}

\section{OpenEdition}

\section{Journals}

Édition électronique

URL : http://journals.openedition.org/monderusse/8283

DOI : 10.4000/monderusse.8283

ISSN : $1777-5388$

\section{Éditeur}

Éditions de l'EHESS

\section{Édition imprimée}

Date de publication : 1 octobre 2015

Pagination : 890-893

ISBN : 978-2-7132-2507-9

ISSN : 1252-6576

\section{Référence électronique}

Catherine Depretto, "Maxim D. Shrayer, I Saw It, Ilya Selvinsky and the Legacy of Bearing Witness to the Shoah », Cahiers du monde russe [En ligne], 56/4 | 2015, mis en ligne le 01 octobre 2015, Consulté le 24 septembre 2020. URL : http://journals.openedition.org/monderusse/8283 ; DOI : https://doi.org/ $10.4000 /$ monderusse. 8283

Ce document a été généré automatiquement le 24 septembre 2020. 


\title{
Maxim D. Shrayer, I Saw It, Ilya Selvinsky and the Legacy of Bearing Witness to the Shoah
}

\author{
Catherine Depretto
}

\section{RÉFÉRENCE}

Maxim D. SHRAYER, I Saw It, Ilya Selvinsky and the Legacy of Bearing Witness to the Shoah, Boston MA : Academic Studies Press (coll. "Studies in Russian and Slavic literatures, cultures and history"), 2013, 340 p.

1 Il'ja Sel'vinskij (1899-1968) fait partie de ces poètes non dénués de talent, assez connus à la période soviétique et tombés dans un oubli presque complet depuis l'effondrement du régime. Proche d'Aseev et de Pasternak, membre du groupe des constructivistes, il s'était rendu célèbre en son temps par des créations audacieuses, hautes en couleur et modernistes. Il paya son tribut au chronos soviétique, y compris par des dithyrambes à Stalin, mais fut aussi l'objet de plusieurs attaques pour manquement idéologique, sans toutefois être personnellement inquiété ${ }^{1}$.

2 Pendant le second conflit mondial, il fut correspondant de guerre à l'exemple d'Erenburg, de Grossman, de Simonov... et entra au parti communiste en 1941. Affecté au front Sud, il suivit l'Armée rouge dans sa brève reconquête de la Crimée en janvier 1942 et fut l'un des premiers à découvrir les tueries massives de civils juifs perpétrées par les Nazis dans les zones occupées. Cela concerne en premier lieu le massacre des 7000 Juifs de Kerč' dans le fossé antichar de Bagerovo², massacre qui, jusqu'à la découverte de Babij Jar, après la libération de Kiev en 1943, est resté en URSS le symbole des tueries massives de Juifs par les Allemands. Sel'vinskij fut d'autant plus bouleversé qu'il était issu d'une famille juive de Crimée (son grand-père était un krymčak). Dès la fin des années 1930, il s'était montré sensible à la persécution des Juifs par les Nazis, à la suite, entre autres, d'un voyage en Allemagne en 1936. Après la 
découverte de Bagerovo, il composa plusieurs poèmes pour témoigner de ce crime, « Ja eto videl [J'ai vu cela] » et un peu plus tard, « Kerč” ».

3 Le livre de Maxim D. Shrayer est consacré principalement à l'histoire de ces poèmes, replacée dans la perspective générale de la trajectoire de Sel'vinskij pendant la guerre et de sa place en tant que témoin de la Shoah. Connu pour s'intéresser aux écrivains juifs de Russie et d'Union soviétique (ouvrage sur l'œuvre du poète Eduard Bagrickij, anthologie de la poésie russe-juive en deux volumes), l'auteur cible ici son investigation sur l'une des questions les plus dramatiques de l'histoire de l'URSS, celle de l'anéantissement par les Nazis des communautés juives des territoires occupés de l'Union soviétique, anéantissement dont il fut possible, dans un premier temps, de parler, mais qu'il fut ensuite impossible de mentionner en tant que tel et ce jusqu'à une période assez récente. Bien qu'il soit difficile d'établir le nombre global des victimes de la Shoah en URSS en raison principalement de la complexité géopolitique des zones occupées, ce chiffre oscille néanmoins entre 2, 5 et 3, 3 millions de personnes, soit au moins la moitié des victimes de la Shoah ${ }^{3}$. C'est dans les territoires occupés de l'Union soviétique, et ce dès l'attaque de juin 1941, que les Nazis se livrèrent aux opérations de tuerie massive de Juifs, confiées principalement aux groupes d'intervention spéciale (les Einsatzgruppen). Ce sont les Soviétiques qui découvrirent les premiers l'ampleur de ce massacre aussi bien sur place que dans les centres de mise à mort, Treblinka, Sobibor, Belzec, Majdanek, Auschwitz-Birkenau... Et si, dans un premier temps, des commissions d'enquête soviétiques furent chargées de rassembler des témoignages et des preuves de ces atrocités, bientôt, il fut difficile d'aborder la question ${ }^{4}$. À partir de 1948, on assista au déchaînement d'un antisémitisme d'État qui se traduisit, entre autres, par l'arrestation et l'exécution des principaux membres du Comité juif antifasciste mis sur pied en 1942, par l'interdiction du Livre noir qui rassemblait une série de témoignages sur les atrocités nazies perpétrées contre les Juifs et par l'anéantissement de la culture yiddish soviétique. L'historiographie de la Shoah en URSS a, de fait, accumulé un retard considérable qu'elle commence à peine à combler aujourd'hui, grâce au travail de quelques équipes de chercheurs, ce dont témoigne, entre autres, la remarquable exposition du Mémorial de la Shoah, "Filmer la guerre " (janvier-septembre 2015) consacrée à ce sujet à travers le prisme du travail des opérateurs de cinéma et des réalisateurs 5 . Mais, malgré ses dimensions considérables, cette question reste l'aspect le moins bien connu de l'anéantissement des Juifs d'Europe ${ }^{6}$.

Si les textes de Sel'vinskij à la mémoire du massacre de Bagerovo étaient connus des spécialistes ${ }^{7}$, personne jusqu'à présent ne s'était livré à une investigation aussi détaillée et aussi précise de cet épisode, comme de la place de Sel'vinskij sur ce sujet (il a également composé "Sud v Krasnodare " 1943 et le poème narratif "Kandava " 1945). À ce titre, l'ouvrage dépasse largement l'étude de cas et ouvre des perspectives intéressantes sur la trajectoire des écrivains soviétiques pendant la guerre. Sel'vinskij n'était pas seul à découvrir l'horreur de Bagerovo : à ses côtés se trouvait Aleksandr Romm (1898-1943), frère du réalisateur de cinéma Mihail Romm, philologue de talent, membre du cercle linguistique de Moscou au début des années 1920 et qui, le premier en Russie, avait entrepris une traduction du Cours de linguistique générale, hélas inaboutie. Dans le poème "Kerč" ", celui-ci est mis en scène sous son nom (et qualifié d'écrivain) ainsi que le critique V.Goffenšefer, également présent : "Nous voilà aussitôt partis. Romm, l'écrivain,/Un photographe, Goffenshefer le critique et moi./Au 
matin nous avons vu un creux/étrangement rayé. C'étaient les mourants/Qui avaient rampé la nuit. ${ }^{8}$ "

5 La biographie d'A. Romm comporte de nombreuses zones d'ombre, en particulier pour la période de la guerre ; affecté à la flottille de la mer Noire comme correspondant de guerre, il se serait donné la mort au front en $1943^{9}$. Le fait qu'il se soit trouvé à Bagerovo n'est pas sans importance : il n'est pas interdit de penser que la découverte du massacre (qui n'a pas dû être un cas unique) l'a profondément marqué. Mihail Romm, en évacuation en Asie centrale pendant la guerre et avec lequel il était en correspondance, s'est très peu livré et n'a pratiquement jamais parlé de son frère. Le cinéaste fut cependant l'un des premiers à se mobiliser contre l'antisémitisme renaissant dans l'administration soviétique à partir de 1943 (lettre à Stalin du 8 janvier 1943) et pendant le dégel, il fut l'un des plus vigilants à s'attaquer aux récidives de l'antisémitisme d'État de la fin des années 1940. Maxim Shrayer ne développe pas ce sujet ; néanmoins son livre donne envie de se pencher sur la trajectoire des frères Romm (appartenant à une famille juive assimilée) et à reprendre l'examen de leurs archives, conservées au RGALI.

En rappelant le rôle de Sel'vinskij comme témoin des massacres de Juifs pendant la guerre, l'auteur cherche à donner une image plus complexe de sa personnalité et de son œuvre. Il met en évidence les sources juives des poèmes, en particulier de « J'ai vu cela » et insiste sur la place de la Crimée dans son œuvre (pas seulement du temps de la guerre). Il élargit également sa recherche aux années qui ont suivi, considérant, entre autres, que la persécution dont Sel'vinskij fut la victime en 1943-1944 pour la publication de "Kogo bajukala Rossija ? [À qui la Russie chantait-elle une berceuse ?] " est la conséquence de ses poèmes sur la Shoah. Sans doute est-il impossible d'établir cette thèse de façon indiscutable. Le sujet renvoie à la question plus générale de la censure et du contrôle politique de la production littéraire et artistique pendant la guerre. S'il est vrai qu'une volonté de cohésion patriotique a permis la réapparition de certaines thématiques (comme le lyrisme), la surveillance ne s'est pas relâchée pour autant, ainsi qu'en témoignent les synthèses (svodki) de la police politique sur l'opinion des écrivains et le durcissement des attaques qui frappent de nombreux créateurs à partir du tournant de 1943 (Aseev, Fedin, Zoščenko, le cinéaste Dovženko...). La condamnation de Sel'vinskij, si violente qu'elle ait été résolution du secrétariat du CC (10 février 1944) contre ce poème, accusé de calomnie contre le peuple russe ${ }^{10}$, interdiction pour lui de repartir au front pour quasiment un an - doit être replacée dans ce cadre général. Le paradoxe est qu'avec ce poème (et d'autres qui s'y rattachent par la thématique comme " Rossija ", " Epizod »), Sel 'vinskij cherchait simplement à exprimer son amour sincère de la Russie. Mais le texte était trop ambigu : les membres du CC s'émurent en particulier de ce que pouvait bien désigner « le monstre que la Russie avait réchauffé en son sein »... et le poète n'avait sans doute pas pris la mesure de la montée du chauvinisme grand-russe : était-ce à un descendant de krymčak de parler de la Russie?

7 Le travail de Maxim Shrayer s'appuie sur de nombreux matériaux, tirés des journaux de l'armée et de la presse locale, sur des fragments toujours inédits du journal de Sel 'vinskij et sur ses lettres à sa femme. Il est richement illustré, comprend une traduction intégrale en anglais des poèmes de Sel'vinskij, "J'ai vu cela » et "Kerč" », ainsi qu'une bibliographie. Tout en redonnant sa place à un poète injustement tombé dans 
l'oubli, il ouvre des perspectives intéressantes sur la trajectoire des écrivains soviétiques pendant la guerre et sur l'histoire de la Shoah en URSS.

\section{NOTES}

1. Sel'vinskij s'est, par exemple, livré à un portrait lyrique de Stalin dans son poème narratif, "Čeljuskiniana » (1937); il a réitéré pendant la guerre avec "Golos Stalina [La voix de Stalin] », "Stalin u mikrofona [Stalin au micro] »..., ce qui ne l'a pas pour autant mis à l'abri des attaques, à ce sujet, cf. Denis Babičenko, éd., "Sčast'e literatury ": Gosudarstvo i pisateli. Dokumenty 1925-1938 [Le bonheur de la littérature: l'État et les écrivains. Documents, 1925-1938], M. : Rosspen, 1997, p. 263-265 ; du même auteur, "Literaturnyj front » : Istorija političeskoj cenzury 1933-1946 gg. Sbornik dokumentov [« le front littéraire »: histoire politique de la censure 1933-1946. Recueil de documents], M. : Enciklopedija rossijskix dereven', 1994, p. 22.

2. Sans doute exagéré, ce chiffre est avancé par Molotov dans sa note du 6 janvier 1942 et sera ensuite repris dans les textes et poèmes de l'époque. Parmi les victimes il y avait également des représentants d'autres nationalités, mais les Juifs constituaient l'immense majorité. Dans cette note, Molotov ne parle pas simplement de " paisibles citoyens soviétiques ", mais mentionne explicitement leur origine juive.

3. Antonella Salomoni, L'Union soviétique et la Shoah, P. : La Découverte, 2008, p. 5-7.

4. Sur cette question, cf. Salomoni, L’Union soviétique et la Shoah.

5. Catalogue de l'exposition, Filmer la guerre 1941-1946. Les Soviétiques face à la Shoah, sous la dir. scientifique de V. Pozner, A. Sumpf, V. Voisin, P. : Mémorial de la Shoah, 2015.

6. Cf. par exemple le récent ouvrage de Georges Bensoussan, Atlas de la Shoah : La mise à mort des Juifs d'Europe, 1939-1945, P. : Autrement, 2014 qui mentionne bien l'action des Einsatzgruppen sur le territoire soviétique, p.35-37, mais ne traite pas de l'extermination des Juifs dans les zones occupées de l'URSS de manière globale. La question est fractionnée entre différentes entrées, avec accent mis principalement sur les Républiques baltes et sur l'Ukraine. Parmi les publications les plus complètes sur ce sujet au niveau de l'information, cf. I.A. Al'tman, éd., Holokost na territorii SSSR : Enciklopedija [L'holocauste en URSS: Encyclopédie], M. : Rosspen, $2^{\mathrm{e}}$ éd., 2011.

7. Ils figurent, par exemple, dans Annie Epelboin, Assia Kovriguina, La littérature des ravins : Écrire sur la Shoah en URSS, P. : Laffont, 2013, p. 193-195.

8. Epelboin, Kovriguina, La littérature des ravins, p. 194.

9. Cf. Catherine Depretto, « Aleksandr Romm (1898-1943), lecteur du Marxisme et la philosophie du langage (1929) ", Bakhtine, Volochinov et Medvedev dans les contextes européen et russe, éd. Bénédicte Vauthier, Slavica Occitania, 25, 2007, p. 399-416.

10. A. Artizov, O. Naumov éd., Vlast' i hudožestvennaja intelligencija. Dokumenty [Le pouvoir et l'intelligentsia artistique : documents], Meždunarodnyj fond Demokratija, 1999, p. 510. D'autres textes officiels avaient précédé cette note finale ; Sel'vinskij lui-même avait été rappelé du front, sommé de s'expliquer devant le comité central lors d'une séance mémorable. 


\section{AUTEURS}

\section{CATHERINE DEPRETTO}

Paris IV 